\title{
Mémoire et politique à Chypre du Nord
}

Les usages des célébrations patriotiques et des manuels scolaires par le nationalisme turc

Memory and Politics in Northern Cyprus: Patriotic Celebrations and School

Textbooks as used by Turkish Nationalism

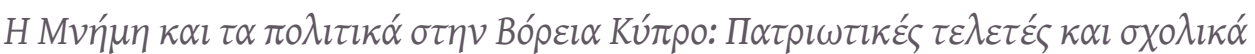

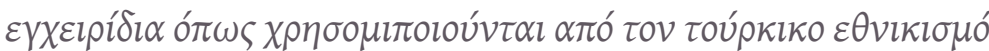

\section{Mathieu Petithomme}

\section{OpenEdition}

Journals

Édition électronique

URL : https://journals.openedition.org/ceb/8611

DOI : $10.4000 /$ ceb.8611

ISSN : 2261-4184

Éditeur

INALCO

Édition imprimée

ISBN : 978-2-85831-229-0

ISSN : 0290-7402

Référence électronique

Mathieu Petithomme, " Mémoire et politique à Chypre du Nord », Cahiers balkaniques [En ligne], 43 |

2015, mis en ligne le 24 juillet 2017, consulté le 06 juillet 2021. URL : http://journals.openedition.org/ ceb/8611 ; DOI : https://doi.org/10.4000/ceb.8611

Ce document a été généré automatiquement le 6 juillet 2021.

Cahiers balkaniques est mis à disposition selon les termes de la Licence Creative Commons Attribution - Pas d'Utilisation Commerciale 4.0 International. 


\section{Mémoire et politique à Chypre du Nord}

Les usages des célébrations patriotiques et des manuels scolaires par le nationalisme turc

Memory and Politics in Northern Cyprus: Patriotic Celebrations and School

Textbooks as used by Turkish Nationalism

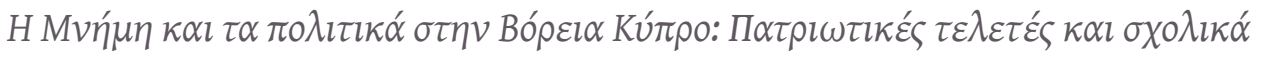

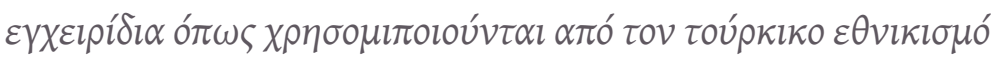

\section{Mathieu Petithomme}

1 L'étude d'une société doit être fondée sur le dévoilement de son " historicité » à partir de ses événements historiques et de ses luttes sociales majeures (Bayart, 2006, 19-61). Mais les représentations du réel et du passé sont sujettes à des luttes idéologiques. La "mémoire collective» d'une nation, transmise par les institutions politiques et éducatives d'État à travers les commémorations patriotiques et l'école, contribue à socialiser un peuple dans une même narration historique sur son passé. Mais elle constitue aussi une vision particulière, qui se fonde le plus souvent sur la narration dominante de l'histoire de cette nation. Dans les sociétés post-conflit, le rapport des citoyens à la « mémoire nationale » demeure un enjeu contentieux qui peut être utilisé politiquement afin de justifier des visions nationalistes de l'histoire. À Chypre, le conflit de 1974 a entraîné la partition de l'île entre la République de Chypre et la « République turque de Chypre du Nord, RTCN » (Kuzey Kıbrıs Türk Cumhuriyeti, KKTC), autoproclamée indépendante depuis le 15 novembre 1983, mais uniquement reconnue par la Turquie. Même si cinq points de passages ont été ouverts en 2003 puis la rue Ledra à Nicosie le 21 avril 2008, le nord et le sud de l'île sont restés physiquement divisés de 1974 à 2003. Le conflit de 1974 a mis fin à une coexistence pluriséculaire entre les Chypriotes grecs et turcs, forgeant des regroupements forcés de population et une territorialisation des identités politiques.

2 Pendant trois décennies, les nationalistes ont dominé les espaces sociaux du nord et du sud, diffusant des visions distinctes de l'histoire du conflit et de la mémoire auprès de 
plusieurs générations de Chypriotes. Impliqué dans un processus de "nationalisation » de la RTCN, entendu comme «la construction d'une identité partagée et d'un sens de l'unité au sein d'une population donnée, à travers l'éducation, la propagande, l'idéologie et les symboles étatiques» (Kolsto, Blakkisrud, 2004, 8), le régime institué par Rauf Denktaş dès 1975, dominé par les anciens combattants de l'« organisation turque de résistance » (Türk Mukavemet Teşkilatı, TMT), s'est fondé sur une propagande exaltant le nationalisme turc, diabolisant les Chypriotes grecs et les «terroristes » de l'EOKA (Ethniki Organosis Kyprion Agoniston). Mais cette vision conflictuelle de la mémoire du conflit a été progressivement remise en cause à la suite des manifestations massives en faveur de la paix et de la réunification de 2002-2003, puis de l'accession au pouvoir du parti républicain turc (Cumhuriyetçi Türk Partisi, CTP) plus favorable à la réconciliation de 2003 à 2009.

Comment les célébrations patriotiques et les manuels scolaires ont-ils été utilisés politiquement par le nationalisme turc pour diffuser une "mémoire nationale " à Chypre du Nord depuis 1974 ? En se fondant sur plusieurs enquêtes de terrain menées de 2012 à 2014, cet article s'intéresse à la politisation de la mémoire du conflit de 1974 à Chypre du Nord. À travers les usages politiques des célébrations patriotiques par le nationalisme turc, il montre la création d'une mémoire nationale « officielle » exaltant la «mère-patrie », la Turquie, et stigmatisant les Chypriotes grecs. Pour autant, il développe la thèse de l'essor d'une "concurrence mémorielle ", entendue comme le développement de visions distinctes de la mémoire et de l'identité collective des Chypriotes turcs (Grandjean et Jamin, 2011). Même si l'espace physique de la RTCN demeure grevé par les symboles du nationalisme turc, les slogans patriotiques, les drapeaux, les statues en l'honneur d'Atatürk, de Fazil Küçük ou d'Ismet Inonü et les monuments en l'honneur des "martyrs", la vision du passé de la propagande d'État connaît une crise relative de légitimité depuis une dizaine d'années, tant l'expérience concrète des Chypriotes turcs est éloignée de l'imaginaire politique des nationalistes louant la partition. Même si plusieurs générations de Chypriotes turcs se sont socialisées dans un environnement nationaliste, ils éprouvent une « distance critique » à l'égard de la propagande d'État. Par leurs pratiques sociales, ils font l'expérience au quotidien du confinement territorial, de l'embargo économique et du caractère artificiel de la souveraineté de la «RTCN». En se fondant sur ce décalage entre le discours politique et l'expérience pratique des acteurs, «le Verbe et la Chair de la société ", l'article illustre les processus d'intériorisation d'une mémoire collective nationaliste et l'existence de visions collectives alternatives (Bayart, 2006, 14).

4 Après avoir présenté le cadre théorique lié aux liens entre mémoire et politique, nous nous intéressons à la politisation de l'enseignement de l'histoire puis aux grandes lignes de la propagande mémorielle du nationalisme turc. Nous étudions ensuite les usages politiques des commémorations et l'affirmation d'un calendrier mémoriel permettant aux nationalistes turcs de diffuser des visions conflictuelles de l'histoire de Chypre et de la mémoire du conflit. La dernière partie revient sur la contestation de ce discours mémoriel nationaliste par leurs homologues chypriotes grecs, mais aussi par les fédéralistes et les pacifiques à Chypre du Nord. 


\section{Mémoire et politique}

5 L'apport du projet des Lieux de mémoire dirigé par Pierre Nora est de montrer la relativité de la connaissance en histoire, en prenant en compte le conflit des interprétations et les usages politiques du passé (Nora, 1984). La réalité historique n'est jamais unilatérale et figée, mais relative et sujette à des interprétations distinctes, à des réévaluations constantes et à des utilisations stratégiques par les historiens eux-mêmes et les acteurs politiques et sociaux. Nora distingue ainsi la "mémoire historique ", plurielle et sujette à des controverses, de la "mémoire collective» d'une nation, à savoir «le souvenir ou l'ensemble de souvenirs, conscients ou non, d'une expérience vécue et/ou mythifiée par une collectivité vivante de l'identité de laquelle le passé fait partie intégrante » (Nora, 1978, 398-401). Maurice Halbwachs montra que la « mémoire collective » a elle-même une histoire, à savoir que les formes socialisées de la présence $\mathrm{du}$ passé à travers les traditions, les symboles et l'enseignement, résultent d'un processus donnant lieu à des conflits d'interprétation puis à une sélection des événements majeurs, dont il s'agit ensuite d'entretenir le souvenir et qui formera la mémoire nationale transmise aux générations futures (Halbwachs, 1997). La mémoire nationale d'une communauté est toujours " politique ", dans la mesure où elle présente une narration historique cohérente pour elle-même et visant à renforcer le lien social.

6 Mais, par le processus même qui donne naissance à sa formulation, cette narration dominante de l'histoire nationale exclut d'autres représentations collectives du passé. En reléguant au rang de "non-événements » certains faits historiques passés, elle dépolitise ainsi d'autres visions alternatives de la mémoire et manifeste l'exercice d'un pouvoir, en ce qu'elle sélectionne les événements et les acteurs qui méritent de faire partie du "récit national », et ceux qui s'en trouvent exclus. Elle résulte de relations de pouvoir et de processus de sélection des cadres d'interprétation historiques. Les acteurs politiques et sociaux dominants imposent ainsi dans l'espace social une certaine vision de la mémoire qui, en étant diffusée par les institutions étatiques, forme le socle d'une mémoire collective au détriment d'autres cadrages (Bachrach et Baratz, 1962, 947-952). Dans l'étude de la construction d'une «mémoire nationale ", il est donc crucial de s'intéresser aux «conditions sociales de production des représentations partagées du passé ", aux mises en récit publiques ou autorisées de l'histoire (Lavabre, 2000, 55). Dans les situations post-conflit comme celle de Chypre, les sociétés se trouvent confrontées à la question cruciale de la gestion politique de la mémoire et du passé.

7 Depuis 1974, les Chypriotes grecs et turcs se sont réorganisés au sein de deux sociétés politiques distinctes, au sein desquelles les nationalistes ont cherché à accroître les différenciations identitaires et les représentations mémorielles exclusives. Les souvenirs des massacres perpétués par l'EOKA-B ou par la TMT, le rôle joué par les généraux grecs dans le soutien à l'enosis et le coup d'État de 1974 ou celui de l'intervention de la Turquie dans la partition de l'île, ont été entretenus sans tenir compte de la mémoire de l'autre (Copeaux et Mauss-Copeaux, 2005). En propageant par l'école, les médias et les célébrations patriotiques, des stéréotypes et des représentations « nationalisantes » de l'histoire contemporaine de l'île largement axées sur l'identification aux « mères-patries » respectives, à l'hellénisme ou au nationalisme turc, les élites politiques de la République de Chypre et de la RTCN ont forgé et diffusé au sein de leurs populations des mémoires collectives antinomiques. Même si des 
«contre-mémoires» collectives peuvent entrer en conflit avec la «mémoire nationale " transmise par les institutions d'État, force est de constater que le souvenir des oppressions passées et le culte aux héros de la lutte nationale entretenus par ces dernières sont souvent contradictoires avec les objectifs de justice, d'apaisement et de réconciliation. Les usages politiques du passé permettent d'illustrer le rôle central de l'imaginaire dans la production du politique. Dans le contexte de Chypre du Nord, nous montrons ainsi comment, à travers la négation de la mémoire de l'autre, la propagande et les célébrations patriotiques, la mémoire a été utilisée politiquement afin de renforcer l'adhésion des Chypriotes turcs au nationalisme turc. Mais des représentations collectives alternatives du passé ont aussi émergé depuis une dizaine d'années, forgeant un processus de concurrence mémorielle et illustrant l'existence d'une mémoire plus intégratrice, moins ethnicisée et plus axée sur le chypriotisme et des aspects civiques et territoriaux.

\section{La propagande mémorielle du nationalisme turc}

8 La propagande mémorielle des nationalistes turcs à Chypre du Nord se fonde sur trois piliers discursifs complémentaires : une simplification exagérée de l'histoire du conflit de 1974, une reconstruction de l'identité nationale des «Turcs» de l'île, et une vision glorifiant le rôle de «sauveur " de l'armée turque et de "bienfaitrice» de la Turquie. D'abord, la partition de 1974 est présentée par les nationalistes comme le point historique culminant de la lutte des « Turcs de Chypre » (Kıbrıs Türkü) pour leur mise en sécurité et le respect de leurs droits dans une nouvelle entité autonome protégée par leur «mère-patrie » (anavatan), la Turquie. Pour Omer Adal, membre du bureau du parti démocrate (Demokrat Parti, DP), «1974 symbolise la libération de notre peuple $»^{1}$. Les partis nationalistes qui ont monopolisé l'espace politique, le parti de l'unité nationale (Ulusal Birlik Partisi, UBP) depuis 1975 et le parti démocrate depuis 1992, demeurent donc très réticents à la remise en cause du statu quo et à la mise en place d'un accord fédéral avec les Chypriotes grecs: dans leur imaginaire politique, le " problème chypriote » a été résolu en 1974, même si la RTCN créée en 1983 demeure un « État illégal » en droit international. Ainsi, pour Cüneyt Küçük, membre du bureau de l'UBP : «Le conflit de 1974 a montré l'agressivité des Grecs à notre égard. Ils nous traitent comme une minorité et veulent nous imposer leurs lois. C'est pourquoi il vaut mieux vivre côte à côte $»^{2}$. Le soutien persistant à l'«indépendance" de la RTCN légitime la division et s'est traduit en 1997, après vingt années de négociations infructueuses, par l'abdication officielle des autorités nord-chypriotes de la recherche d'une solution fédérale, au profit d'une posture confédérale.

De plus, la propagande nationaliste cherche à reconstruire une identité nationale "turque " en présentant les "Grecs» de façon stéréotypée et comme des boucs émissaires, tout en niant l'existence d'une identité « chypriote ». Rauf Denktaş déclara lui-même à la télévision publique (BRT 1) en juin 2002: «Il n'existe pas de Chypriotes sur cette île. Le seul vrai Chypriote, c'est l'âne de Chypre $»^{3}$. Les nationalistes mettent ainsi en avant l'existence de "deux peuples ", les "Turcs » et les "Grecs ", pour légitimer la division. À l'inverse, la position officielle de la République de Chypre est d'évoquer un seul peuple de Chypre dans les négociations internationales pour légitimer la réunification. Au nord, les nationalistes distinguent «les Turcs» et «les Grecs » et parlent au nom du «turquisme » (Türklük), de la défense des Turcs contre 
l'agression des "Grecs", un discours qui est le miroir de celui des nationalistes chypriotes grecs qui présentent l'hellénisme comme un recours contre l'« expansionnisme turc» (Tourkikos Epektatismos) (Papadakis, 1993, 141). Kemal, un militaire turc, parle ainsi des Chypriotes turcs comme de ses " concitoyens ", en écho à la propagande nationaliste qui les présente comme partie prenante de la même «nation» turque : «La Turquie doit défendre ses concitoyens contre les attaques des Grecs qui voulaient les chasser de l'île. Nous les militaires, nous sommes ici pour les défendre $»^{4}$.

De 1974 à 2003, des années où les nationalistes ont dominé l'ensemble des institutions politiques (la Présidence, le Parlement et le gouvernement), ils se sont efforcés de distiller à travers l'école, les médias et les célébrations patriotiques, une propagande fondée sur un "affect de remerciement» glorifiant l'armée turque comme une «bienfaitrice » qui aurait «sauvé » les Chypriotes turcs de l'ennemi (Navaro-Yashin, 2012, 89). La Turquie les aiderait "par solidarité désintéressée envers un peuplefrère $~_{5}^{5}$. Çetin Uğural et Ferdi Sabit Soyer parlent ainsi d'une "économie de la gratitude » (şükran Ekonomisi), où un discours politique qui exagère le rôle de l'« aide turque » domine l'espace public, en essayant de persuader la population que tant le maintien de la présence de l'armée turque que le soutien de la Turquie sont indispensables (Uğural et Soyer, 1998). Les propos de représentants de l'extrême-droite et de militaires turcs illustrent bien cette justification du rôle "indiscutable " de l'armée turque comme "garante» de la sécurité des Chypriotes turcs. Pour Mehmet Enginar, responsable de la section des Loups Gris (Ülkü Ocaklari) de Kyrenia :

Il n'est pas possible de critiquer la présence de l'armée. Elle agit pour le bien du peuple. Elle est au-dessus des querelles politiciennes, son rôle ne peut pas faire l'objet de débats politiques. Elle est là pour préserver l'intérêt national des Turcs de cette île ! C'est une question qui porte atteinte à la sécurité de l'État ${ }^{6}$.

11 Pour Huseyin, un militaire turc, la présence durable de l'armée turque se justifierait non pas par ses intérêts et sa volonté de puissance et de domination, mais par l'impossible entente entre les Chypriotes grecs et turcs. Elle ne serait présente à Chypre que « par nécessité » et altruisme pour soutenir et porter secours aux Chypriotes turcs :

Si les Chypriotes turcs n'avaient pas été en danger, l'armée turque n'aurait pas besoin d'être là, et moi je resterais tranquillement en Turquie. Mais les Chypriotes turcs ont besoin de nous, car les Grecs leur veulent du mal. Ils ne veulent pas d'eux ici à Chypre, ni de la RTCN. L'armée turque voudrait bien retourner en Turquie, mais elle ne peut pas $^{7}$.

Cette propagande a été distillée parmi la population grâce aux institutions d'État, dans une société séparée tant physiquement que psychologiquement de l'autre partie de l'île de 1974 à 2003. Pour Alpay Durduran, un dirigeant politique historique du mouvement pour la paix, désormais secrétaire pour les relations internationales du parti de la Nouvelle Chypre (Yeni Kibris Partisi, YKP), les nationalistes promeuvent une politique de turquification à travers l'usage des médias et du système éducatif :

L'intégration de la RTCN à la Turquie est soutenue à tous les niveaux. Les principaux médias dépendent tous de la Turquie, cela permet de renforcer son influence. De même, le système éducatif est calqué sur celui de la Turquie, plutôt que de mettre en avant notre propre système avec notre vision des choses. C'est particulièrement flagrant dans l'enseignement de l'histoire au lycée par exemple, il faut voir ce que l'on apprend aux élèves ! L'armée turque protectrice des Chypriotes turcs ! 
En maniant indistinctement la peur et la menace, en présentant la partition comme un progrès social, et l'enfermement des Chypriotes turcs dans une entité non reconnue comme un sacrifice nécessaire à leur cause, les nationalistes ont forgé un contexte politique où la liberté de pensée demeure partielle. Aujourd'hui encore, il est risqué de s'opposer publiquement à la présence de l'armée. Lorsque les fédéralistes veulent manifester contre «l'occupation " (İsgal) et en faveur de la réunification, il n'est pas rare qu'ils n'obtiennent pas d'autorisation au motif de possibles «troubles à l'ordre public ». Lors des mouvements sociaux de 2002-2003, ils ont souvent été contraints de manifester sans autorisation, ce qui n'a pas empêché des protestations massives contre le régime sclérosé de Rauf Denktaş. Mais sur le long terme, cette contrainte policière, cette «ligne rouge » à ne pas franchir, limite les perspectives de mobilisation des citoyens, puisque nombreux sont ceux pour qui le « coût " personnel de l'engagement (arrestations, pressions au travail, etc.) demeure trop important (Olson, 2011). Autant les affrontements entre les acteurs politiques chypriotes turcs sont parfois très véhéments, autant les critiques à l'égard de l'armée turque et de la Turquie font l'objet d'un «tabou » qui réduit l'espace discursif, et contraint implicitement «ce que l'on peut et ce que l'on ne peut pas dire » dans les échanges politiques et publics (Bourdieu, 1982).

\section{La politisation de l'enseignement de l'histoire}

Dans le rapport à la mémoire nationale, l'enseignement de l'histoire à Chypre constitue un enjeu crucial de la socialisation politique des Chypriotes grecs et turcs. Au sein des communautés où coexistent différentes allégeances nationales, l'école constitue un environnement idéal pour transmettre un message nationalisateur. L'histoire est souvent utilisée afin de diffuser une narration se fondant sur les souffrances et les oppressions passées d'une communauté afin de légitimer un projet politique de construction nationale. Dans la création de "mythes" nationaux et dans les interprétations des «faits » historiques, l'éducation et l'enseignement de l'histoire peuvent être instrumentalisés afin de maintenir des stéréotypes à l'égard de l'« autre » en encourageant la méfiance réciproque, ou servir au contraire de fondement à la coexistence pacifique et à la compréhension mutuelle (Coakley, 2004, 533). En Grèce et en Turquie, l'enseignement de l'histoire a ainsi été utilisé par les idéologies nationalistes. Pour Hercules Millas, "les Grecs et les Turcs ont été éduqués pour devenir des antagonistes et des opposants. Depuis des générations, ils ont été nourris d'idéologies agressives, de stéréotypes à l'égard de l'autre, d'informations unilatérales, de distorsions et d'exagérations historiques" (Millas, 1991, 23). En Grèce, l'enseignement de l'histoire est strictement encadré par l'État et soumis aux pressions des cercles nationalistes, qui ont obligé en 2002 le ministère de l'Éducation à retirer le qualificatif « d'organisation promouvant un nationalisme ultra-conservateur » au sujet de l'EOKA chypriote. Loris Koullapis a aussi montré comment les milieux nationalistes ont de même poussé le gouvernement en septembre 2007 à renoncer à une refonte des manuels du primaire, au motif que la réforme aurait cherché à «imposer une déhellénisation »(Koullapis, 2002a, 290).

15 À Chypre, le système ottoman des millets octroya l'autonomie en matière religieuse et d'éducation aux orthodoxes et aux musulmans de l'île, qui furent éduqués au sein de leur communauté de 1571 à 1878 . Le sentiment d'appartenance "chypriote » fut 
ensuite promu par le colonisateur britannique en cherchant à résister à l'importation du nationalisme grec sur l'île, d'abord sous le protectorat (1878-1914) puis lorsque Chypre devint une colonie de la Couronne (1914-1960). Mais pour Naday Morag, en maintenant le dualisme éducatif, l'administration coloniale britannique continua à " socialiser les jeunes Chypriotes en tant que Grecs ou Turcs plutôt que de favoriser le développement d'une identité civique chypriote» (Morag, 2004, 605). La République bicommunautaire indépendante de 1960 consacra formellement l'autonomie de chaque communauté en matière d'éducation. Une identité chypriote commune n'a donc jamais été réellement promue de façon désintéressée par les institutions d'État, contrairement aux patriotismes et aux allégeances respectives à la Grèce et à la Turquie (Theophylactou, 1995, 58).

16 Depuis la partition de 1974, la prévalence de perspectives "ethno-nationalistes" opposées a eu pour effet de systématiquement présenter l'île comme partie prenante de la Grèce ou de la Turquie, justifiant les interprétations nationalistes de l'histoire prônant l'union avec la Grèce (enosis) ou la partition (taksim) (K1z1lyürek, 2002, 432-435). Aujourd'hui encore, le système éducatif de la République de Chypre présente les Chypriotes comme "des sujets de la nation grecque» imbriqués dans l'histoire de l'hellénisme, en ignorant quasiment la contribution des Chypriotes turcs à l'histoire de l'île. Malgré le travail d'un "Comité pour une réforme de l'éducation » établi sous la présidence de Tássos Papadópoulos (2003-2008), aucune réforme n'a vu le jour (Koullapis, 2002b, 407). Il a fallu attendre la présidence de Dimítris Khristófias de 2008 à 2013, ex-secrétaire général de l'AKEL de 1988 à 2009, pour qu'une réforme soit engagée afin de produire des manuels d'histoire qui encouragent la compréhension de l'autre et la coexistence pacifique en favorisant une vision historique qui intègre les deux composantes du « peuple chypriote ». Étienne Copeaux a bien montré que tant en Turquie qu'en RTCN, il n'existe pas seulement un contrôle officiel sur « les contenus de l'enseignement de l'histoire », mais « des directives sont aussi publiées afin d'expliquer le sens politique et idéologique au sein duquel chaque professeur doit fondre ses leçons » (Copeaux, 2002, 398).

17 En RTCN, l'histoire doit obligatoirement être enseignée, dans le public et le privé, sur la base des manuels approuvés par le «ministère de l'Éducation nationale et de la culture » (Vural et Özuanık, 2008, 141). Jusqu'en 2004, les cours « d'histoire de Chypre » portaient en fait sur "l'histoire des Turcs de Chypre» et non sur «les réseaux complexes et multiséculaires de relations bicommunautaires sous les règnes ottomans et britanniques » (Canefe, 2002,383-396). Deux manuels d'histoire, réédités à plusieurs reprises, étaient utilisés depuis 1974: "L'histoire de Chypre " au collège, rédigé par Vehbi Zeki Serter, un historien chypriote turc et député de l'UBP nationaliste; et "L'Histoire de la lutte des Turcs de Chypre " au lycée (Zeki, 2002 ; Zeki et Fikretoğlu, 2002). Ces manuels ne parlaient pas de l'histoire sociale commune des Chypriotes. Ils prédisposaient les élèves à percevoir leur identité « turque » comme incompatible avec celle des Chypriotes grecs, avec qui la coexistence était présentée comme impossible. Dans leur étude empirique détaillée de ces manuels, Yücel Vural et Evrim Özuanık montrent que le qualificatif d'«identité chypriote» et l'existence même des "Chypriotes » étaient niés, en préférant les notions de « Turcs » et « Grecs » de Chypre, afin d'accroître la différenciation de deux communautés présentées comme « ethniques » et «différentes par la religion, la langue et la race» (Vural et Özuanık, 2008, 142-146). L'idée qu'«il existe deux peuples séparés » à Chypre était présentée comme « une réalité » impliquant «le droit à l'autodétermination et la partition de 
l'île ». Chypre serait une "extension géologique naturelle de l'Anatolie », de telle sorte que les Chypriotes turcs devraient être «loyaux envers les principes, la révolution d'Atatürk » et la «lutte nationale ». Il était défendu que «la destinée des Chypriotes turcs dépend de la Turquie », caractérisée comme "leur mère-patrie ». Les "Turcs de Chypre " étaient présentés comme "des descendants des immigrants turcs qui s'installèrent après la conquête de Chypre par les Ottomans en 1571 ", ayant conservé "leur histoire, leur langue et leur culture", donnant ainsi une vision d'une pureté ethnique immuable. Au contraire, "les Grecs qui vivent à Chypre aujourd'hui » ne seraient «pas de vrais Grecs, mais résultent des mélanges ethniques entre les différentes communautés qui ont conquis l'île dans l'histoire ». Ces manuels diffusaient les idées d'une conflictualité historique et d'une allégeance nécessaire "aux mèrespatries ». Pour Izzet Izcandir, cette politisation de l'enseignement de l'histoire a permis aux nationalistes de modifier les perceptions de plusieurs générations de Chypriotes turcs :

On a effacé tout ce qui faisait référence à Chypre, à la culture chypriote et au passé commun. On ne parle quasiment plus des "Chypriotes », et on enseigne aux enfants que leur nation c'est la Turquie et que leurs ennemis, ce sont «les Grecs». En histoire, mais aussi en littérature, on apprend aux enfants une histoire qui est celle de la Turquie, mais qui n'est pas la leur, celle de Chypre, leur pays. Pour la littérature, c'est la même chose, on n'étudie que la littérature et la civilisation turque, mais pas la littérature "chypriote», qu'elle soit chypriote grecque ou turque. On a imposé un véritable lavage de cerveau aux enfants depuis presque 30 ans maintenant! Donc imaginez, cela fait trois générations d'enfants qui sont « éduqués »si on veut, dans le rejet des Chypriotes grecs et la croyance que leur nation, celle qui les protège, c'est la Turquie! C'est une propagande d'État très puissante !

L'affaiblissement du discours nationaliste et l'« effet catalyseur» de la perspective d'adhésion à l'UE ont favorisé une alternance historique en 2003, à travers la formation d'un gouvernement dirigé par le parti républicain turc (CTP). En 2004, des commissions de travail furent formées par le ministère de l'Éducation afin de réviser les manuels, ce qui déboucha en 2006-2007 sur la publication de trois nouveaux manuels pour le collège ("Histoire de Chypre»), et de deux nouveaux manuels pour le lycée (" Histoire de Chypre» et "Histoire des Turcs de Chypre»). Ces manuels scolaires proposent une narration nouvelle du territoire, de l'histoire et de l'identité. Ils mettent en avant une identité «chypriote» commune, encouragent une vision plus égalitaire avec les Chypriotes grecs et une loyauté plus forte à l'égard de l'île, jugent péjorativement le conflit et reconnaissent le caractère commun de leurs souffrances passées (Vural et Özuanık, 2008, 143). Ils évoquent "l'identité chypriote des Chypriotes turcs » et se réfèrent à la catégorie des "Chypriotes " pour caractériser l'ensemble de la population de l'île. Ils se référent fréquemment aux concepts de "Chypriotes turcs" et de "Chypriotes grecs ", et insistent sur la responsabilité des acteurs externes, notamment la Grande-Bretagne et la Turquie, dans la partition. L'origine historique de Chypre est datée de la formation du mont Trodos il y 200 millions d'années, « qui forma une petite île », et non d'un détachement géologique d'une partie de l'Anatolie (POST, 2007, 17).

19 Chypre est considérée comme "la patrie de toute la population vivant sur l'île", incluant les «Chypriotes grecs et les Chypriotes turcs» (POST, 2007, 15). Les manuels évoquent la difficulté historique de l'île de maintenir son indépendance face aux invasions et aux puissances extérieures (Vural et Özuanı, 2008, 143). Les points communs entre les deux communautés sont beaucoup plus mis en avant: «même si 
leur religion et leur langue diffèrent, les Chypriotes grecs et les Chypriotes turcs ont vécu ensemble pendant des siècles. Ils aiment tous Chypre et considèrent l'île comme leur "patrie" " (Vural et Özuanık, 2008, 144). Des exemples de coexistence pacifique sont donnés. Plutôt que de rejeter la responsabilité du conflit de 1974 sur la communauté chypriote grecque comme le faisaient les anciens manuels, les nouveaux manuels expliquent les causes sociologiques et géopolitiques des affrontements et insistent sur le rôle néfaste des nationalismes grec et turc. Les nouveaux manuels expliquent que « le nationalisme de l'enosis est exclusif. Pour cette raison, il empêcha le développement d'un nationalisme chypriote fondé sur l'unification des deux communautés » (Vural et Özuanık, 2008, 145). Ils reconnaissent aussi « les souffrances passées de l'ensemble des Chypriotes ", en donnant des exemples de civils chypriotes grecs persécutés par les milices de la TMT et de civils chypriotes turcs par l'EOKA-B.

L'alternance historique de 2003 a ainsi débouché, dans le champ de l'enseignement, sur une refonte des manuels scolaires d'histoire proposant une redéfinition des frontières de l'identité et de la nation. Le gouvernement de centre-gauche proposa une vision moins conflictuelle, en « démilitarisant » l'enseignement de l'histoire et en proposant une vision civique et plus ouverte de l'identité, en mettant en avant les points communs, la coexistence pacifique multiséculaire et les souffrances partagées des Chypriotes grecs et turcs. Conscient de l'imprégnation dans les mentalités de l'association nationaliste entre «identité chypriote» et "Chypriotes" avec la République de Chypre et les Chypriotes grecs, le gouvernement a cherché à promouvoir une identité chypriote turque qui serait partie prenante d'une identité chypriote commune. Mais ce processus de redéfinition identitaire connait aussi des limites. L'hymne national et le drapeau turc demeurent présentés comme des symboles de l'« identité chypriote turque »; certains événements historiques mettent plus en avant les souffrances des Chypriotes turcs et passent parfois sous silence celles des Chypriotes grecs; l'intervention de l'armée turque de 1974 demeure présentée comme une "opération de paix " (Papadakis, 2008, 10). La refonte des manuels scolaires constitue toutefois une avancée positive afin de promouvoir la réconciliation, le dialogue et une vision plus nuancée de l'histoire. Mais ce processus demeure éminemment contesté par les nationalistes, qui défendent comme Saffet Nadiri, qu'il s'agit "d'une entreprise politique de falsification de l'histoire » et qui veulent revenir aux anciens manuels ${ }^{10}$. En mai 2014, le directeur de la BRT, la télévision publique nationale, a d'ailleurs déclaré que le terme "Chypriote turc » ne devait plus être utilisé à la télévision, imposant aux journalistes des chaînes publiques de parler plutôt des « Turcs de Chypre »"

\section{Fêtes nationales et commémorations}

\section{L'affirmation d'un calendrier mémoriel nationaliste}

Depuis 1974, les nationalistes ont inséré leur communauté politique dans le calendrier mémoriel de la Turquie. En présentant les célébrations patriotiques d'un autre État comme partie prenante de l'histoire des Chypriotes turcs, les nationalistes « mettent en scène » leur allégeance à la Turquie et revendiquent leur appartenance à la nation turque. L'opposant Murat Kanatli, secrétaire général du parti de la Nouvelle Chypre (Yeni Kıbrıs Partisi, YKP) est très critique de ces cérémonies, qui permettraient aux nationalistes de légitimer la présence de l'armée turque : 
Pour montrer sa toute-puissance, huit cérémonies sont organisées chaque année. Les routes sont bloquées et l'armée défile pour apparaître comme la "protectrice " des Chypriotes turcs: le 20 juillet, la date "anniversaire» de l'occupation; le 13 août pour célébrer la «victoire» de la Turquie; le 29 octobre pour l'établissement de la République turque ou encore le 15 novembre pour la fondation de la RTCN. Toutes ces cérémonies sont utilisées pour présenter l'armée turque comme une force de défense de la RTCN, alors que c'est une force occupante de l'île de Chypre ${ }^{12}$.

"Enfant-patrie " (yavru vatan) de la République de Turquie, la RTCN se doit donc selon les nationalistes de célébrer le "Jour des enfants et de la souveraineté nationale " (Ulusal Eğemenlik ve Çocuk Bayramı) le 23 avril, qui commémore la première réunion à Ankara en 1920 de la Grande Assemblée nationale, premier parlement de l'État de Turquie, une fête nationale dédiée par Atatürk aux enfants, «futurs de la nation ». La fête nationale turque du 19 mai 1919 est aussi célébrée à Chypre du Nord. En rappelant l'offensive d'Atatürk à Samsun en réaction au déploiement des Grecs en Asie Mineure le 15 mai 1919, elle permet de mobiliser les ressentiments à l'égard des Grecs. À Chypre du Nord, cette fête a une double signification en tant que «Journée de la jeunesse et des sports » (Gençlik ve Spor Bayramı) et de la "Mémoire d'Atatürk» (Atatürkü Anma). Ce stratagème mémoriel permet aux nationalistes de mobiliser les jeunes autour d'activités sportives, tout en célébrant la mémoire d'Atatürk et en scellant dès leur plus jeune âge leur allégeance symbolique à la Turquie. Le 23 avril, l'« Anniversaire de la Grande Victoire » (Büyük Zafer Bayramı) contre l'armée grecque du 30 août 1922 et le "Jour de la République » (Cumhuriyet Bayramı) qui célèbre la fondation de la République de Turquie le 29 octobre 1923, sont autant de jours fériés en Turquie et en RTCN, commémorés de façon enthousiaste par les nationalistes à Chypre du Nord (Papadakis, 1993, 153).

L'ensemble de ces festivités, comme celle de la mort d'Atatürk (Atatük'ün ölümü) le 10 novembre 1983, ne se réfèrent en aucune manière à un calendrier mémoriel "chypriote » qui serait lié aux événements et à l'histoire insulaire, et médiatisent l'importation à Chypre des célébrations patriotiques de la République de Turquie. Le calendrier mémoriel chypriote turc et la rhétorique nationaliste sont fortement dominés par un ethos de reconnaissance, une " économie de la gratitude » : la Turquie est présentée comme un État " protecteur ", dont les forces armées auraient "sauvé " les Chypriotes turcs de l'extermination (Uğural et Soyer, 1998). La semaine du 13 au 20 juillet est ainsi utilisée par les nationalistes afin d'organiser des "activités de gratitude » (şükran Etkinlikleri) qui culminent par le «Jour de la paix et de la liberté » (Bariş ve Özgürlük Bayraml), qui commémore l'intervention militaire de l'armée turque à Chypre du 20 juillet 1974, officiellement nommée "l'Opération de paix à Chypre " (Kıbrıs Bariş Harekâti). Des défilés et des cérémonies militaires sont organisés sur l'ensemble du territoire. Pour affirmer sa puissance, l'armée turque n'hésite pas à sortir les chars des casernes, ce qui suscite la curiosité des enfants, la fierté des nationalistes, mais engendre aussi un certain malaise.

\section{La célébration de la mémoire des « martyrs »}

Mais le calendrier mémoriel « officiel» de la RTCN inclut aussi des célébrations qui commémorent la lutte des combattants de la TMT, les victimes et les martyrs chypriotes turcs du conflit. La date du 1er août, le «Jour de la résistance commune », a 
ainsi été choisie par les autorités pour commémorer tant la "Conquête de Chypre " (Kıbris'in Fethi), la prise de Famagouste par les Ottomans en 1571, la "naissance de la TMT » (TMT Doğusu) en 1958 et la «Fondation de nos forces de sécurité » (Güvenlik Kuvvetlerımizin Kuruluşu) en 1976 (Papadakis, 2003, 263). Certes, le 1er août 1571, sous les ordres de Selim ii, les 61000 hommes de l'armée régulière (Kapikulu) et des unités provinciales (Eyalet Askerleri) ottomanes dirigées par l'amiral Piyale Pasha s'emparèrent de Famagouste ce qui, après la prise de Nicosie le 25 juillet 1570 et la décapitation du gouverneur vénitien Nicolo Dandolo, scella la prise de Chypre par des Ottomans (Blondy, 1998, 21). Mais selon Michael Attalides, «les circonstances historiques de la naissance de la TMT demeurent obscures » (Attalides, 1979, 46). L'« organisation turque de défense» (Türk Mukavemet Teşkilatı) émergea en 1958 à partir du groupe paramilitaire Volkan, mais rien n'indique que ce fut le 1er août, pas plus que les «forces de sécurité » chypriotes turques ne furent créées en 1976 puisque les milices de la TMT jouèrent ce rôle dans les enclaves dès 1963 avant même la proclamation de l'« État turc fédéré de Chypre » en 1975 (Navaro-Yashin, 2012, 89). En réinventant l'histoire et en incorporant l'ensemble de ces événements historiques distincts dans le cadre d'une même commémoration, les nationalistes cherchent à les présenter comme autant de «bornes mémorielles d'un même processus de construction nationale (Petithomme, 2015).

Dans la création d'une mémoire nationale, les célébrations en l'honneur des « martyrs » de la cause chypriote turque jouent un rôle essentiel. Sur les ronds-points et à l'entrée des villes et des villages, de nombreux monuments célèbrent les combattants de 1974. À Gönyeli, le "monument de la lutte nationale et de la liberté ", composé de quatre piliers en pierre et de statues en bronze, met en scène des civils et des combattants qui luttent et marchent vers la liberté. À Famagouste, un monument analogue fait l'apologie de la lutte armée. Lors des cérémonies du 23 avril, du 1er août ou de l'« indépendance» de la RTCN le 15 novembre 1983, les hommages aux martyrs entretiennent une mémoire belliqueuse du conflit. La mémoire des massacres de 126 civils par l'EOKA-B le 14 août 1974 à Muratağa (Maratha), Atlilar (Santalaris) et Sandallar (Aloda), est souvent évoquée afin de condamner la «barbarie» des Chypriotes grecs avec qui toute coexistence serait donc impossible. Cela déshonorerait le sacrifice des héros de la lutte nationale. Dans un entretien au journal Kibris, Hakki Atun (DP), Premier ministre de 1993 à 1996, déclara ainsi le 2 avril 1997 : « Notre peuple est aujourd'hui encore un bouclier contre les idéaux de l'EOKA. Ces assassins cherchent l'extermination des Turcs de Chypre. Mais grâce à notre "mère-patrie" nous pouvons désormais vivre en paix en RTCN $»^{13}$.

La «semaine de la lutte et de la mémoire des martyrs" (şehitleri Anma ve Mucadele Haftası) réfère aux événements du « Noël Sanglant » (Kanl Noel) du 21 décembre 1963, lorsque des affrontements intercommunautaires éclatèrent suite à la révélation $\mathrm{du}$ projet de révision constitutionnelle de Makarios et du plan Akritas pour la mise en place de l'enosis (Dodd, 1993, 45). Lors des célébrations du $1^{\mathrm{er}}$ août et du 21 décembre, le gouvernement, les forces de police, les anciens combattants de la TMT et les nationalistes paradent en arborant des drapeaux turcs et chypriotes turcs de même que le drapeau bleu des Loups Gris symbole du panturquisme. Le slogan « Nous n'oublions pas » (Unutmayacağiz) est scandé, en miroir du slogan chypriote grec «Je n'oublie pas » (Den Xehno), pour rappeler la "brutalité » des Chypriotes grecs, «le sacrifice des martyrs » et la " persistance de la menace », selon les discours publics nationalistes ${ }^{14}$. 
Les célébrations de la «Révolution grecque contre les Turcs » du 25 mars 1921, fête nationale en Grèce et dans la République de Chypre, de même que celles du $1^{\text {er }}$ avril 1955 présentées par les Chypriotes grecs comme le début de la «lutte de libération nationale » de l'EOKA pour l'union avec la Grèce, sont instrumentalisées par les nationalistes du nord pour montrer selon eux l'impossibilité de faire confiance aux Chypriotes grecs qui honorent toujours les « tueurs de l'EOKA» (Papadakis, 2003, 265).

\section{La contestation de la mémoire nationaliste}

La vision de l'histoire proposée par les nationalistes constitue une "narration de l'identité » qui replace certains événements révélateurs dans une histoire plus vaste d'oppression et de lutte (Anderson, 2006, 205). Dans le contexte chypriote, ce processus d'«invention" d'une mémoire nationale demeure très conflictuel, car il met en exergue certains événements et en passe d'autres sous silence, en cherchant à donner "une continuité et une cohérence » à la narration historique proposée (Hobsbawn, 1992, 11). Les partis de gauche au nord, le CTP et le TKP, collaborent avec l'AKEL, le parti communiste chypriote grec, et fournissent des interprétations plus inclusives de l'histoire de Chypre, moins communautarisées et moins dépendantes des histoires de la Grèce et de la Turquie. À l'inverse, les partis nationalistes au nord (UBP et DP) comme au sud (DISY), partagent une antipathie mutuelle. Le calendrier mémoriel proposé par les nationalistes du nord est ainsi contesté par les nationalistes du sud, qui proposent une histoire présentant Chypre comme la "Grande Île au cœur " (Akritiki Megalonissos) de l'hellénisme, les Chypriotes comme une composante du "peuple grec» et les Chypriotes turcs comme une « minorité ».

\section{La mémoire des nationalistes chypriotes grecs}

Le Rassemblement démocratique (DISY) qui attire environ un tiers de l'électorat chypriote grec depuis 1974, constitue « le point de ralliement des éléments les plus conservateurs et droitiers à Chypre » (Coufoudakis, 1983, 115). Il fallut ainsi attendre le 2 octobre 1989 pour que le DISY brandisse pour la première fois des drapeaux de la République de Chypre et non de la Grèce pour commémorer l'« indépendance » (Mavratsas, 1997, 717-737). Son projet politique est la continuation de la lutte des «combattants de l'EOKA », re-labellisée après 1974 comme «la lutte pour l'indépendance» et commémorée le $1^{\mathrm{er}}$ avril 1955, même s'il s'agissait d'abord d'une lutte pour l'union avec la Grèce (enosis) (Papadakis, 2003, 256). DISY constitua un refuge pour les ex-membres de l'EOKA-B, et réincorpora lors de son accession au pouvoir en 1999, «les 62 » employés du gouvernement qui participèrent au coup d'État du 15 juillet 1974. Ceci explique que le DISY refuse de commémorer cette date et soutient de façon très passive la célébration officielle de «l'indépendance de la République de Chypre » en 1960, fêtée le 1er octobre, perçue comme le symbole de l'échec de l'union avec la Grèce.

La commémoration organisée par le NEDISY, le mouvement de jeunes du parti, et l'association des combattants de l'EOKA en l'honneur de la mort du Général Grivas le 27 janvier 1974, souligne la vénération de la lutte armée de l'EOKA. Le personnage de Grivas joue le rôle d'une "figure héroïque fonctionnelle " pour le nationalisme grec à Chypre (Casquete, 2009, 139). Le fait que le DISY passe sous silence le 15 juillet illustre une volonté de nier l'importance historique du coup d'État de 1974, considéré comme 
secondaire et source de division avec les Grecs. Le parti commémore plutôt l'« invasion turque barbare » du 20 juillet 1974, la "Révolution contre les Turcs » (25 mars 1821) et le «Jour du Non » (28 octobre 1940), lorsque le dictateur grec Metaxás refusa de s'allier avec l'Italie, illustrant une volonté d'insérer le calendrier mémoriel de Chypre dans celui de la Grèce en mettant l'accent sur le conflit helléno-turc et la figure du " Turc » comme ennemi séculaire. L'histoire proposée par le DISY est celle de la résistance de l'hellénisme pensée en opposition à l'« expansionnisme turc » (Tourkikos Epektatismos).

\section{La contre-mémoire des fédéralistes et des pacifistes} une célébration centrale du calendrier mémoriel national : historiquement proclamée le 16 août 1960, il fallut attendre le gouvernement Vasiliou dirigé par l'AKEL pour que l'indépendance soit commémorée à partir de 1979, et que sa célébration soit transférée par décret le $1^{\mathrm{er}}$ octobre, qui devint un jour férié. Pour l'AKEL, la commémoration de la mort du Général Grivas par le DISY le 27 janvier constitue une falsification de l'histoire, en présentant comme un héros un homme d'extrême-droite responsable du conflit et qui a fait assassiner des militants de gauche et des civils dans les années 1970. Les associations de «combattants résistants pour la démocratie » de l'AKEL ont donc organisé à partir des années 1980 une "contre-commémoration" le 9 janvier en l'honneur du Président Makarios (que l'EOKA-B chercha à assassiner), jour de sa fête dans le calendrier grec orthodoxe, afin de célébrer cette figure historique consensuelle de la société chypriote grecque, de même que les « combattants résistants » contre les putschistes de 1974 (Papadakis, 1998, 153).

31 La même logique d'opposition symbolique à la mémoire nationaliste proposée par le DISY prévaut dans l'organisation de l'« Anniversaire sombre du Coup et de l'invasion " organisée le 15 juillet, qui voit les sympathisants de l'AKEL défiler en scandant le slogan «Le peuple n'oublie pas les fascistes et les tanks» (Papadakis, 2003, 261). L'AKEL présente aussi le «Jour du Non» du 28 octobre 1940 contre l'Allemagne nazie, comme une célébration antifasciste plus générale, dirigée aussi à la junte grecque et l'EOKA-B. L'AKEL propose une mémoire nationale non pas fondée sur l'hellénisme, mais sur l'histoire commune des Chypriotes grecs et turcs. Le parti commémore enfin le meurtre le 11 avril 1965 de deux militants de l'AKEL, Mishaoulis (Chypriote grec) et Kavazoğlü (Chypriote turc) par des combattants de la TMT, qui symbolise la lutte démocratique conjointe de membres des deux communautés contre les extrémismes. Chaque année, EDON, son mouvement de jeunesse organise autour de cette date une semaine de rencontres bicommunautaires et de mobilisations en faveur du "Rapprochement " (Epanaproseggisi), qui devint une politique officielle après 1974, en remémorant la coexistence pacifique passée.

nord, les partis de gauche contestent aussi les références aux histoires de la Grèce et de la Turquie. Ils ont souvent été exclus des commémorations officielles jusqu'aux années 2000. Le parti républicain turc (CTP) prône ainsi une mémoire de la résistance démocratique lors de la "Commémoration des martyrs de la démocratie " (Demokrasi şehitleri Anma) le 24 avril 1962, qui a la même signification que celle des « combattants résistants pour la démocratie » de l'AKEL. Le CTP remémore les assassinats de Gurkan et Hikmet, deux journalistes chypriotes turcs assassinés par la TMT qui défendaient la coopération avec les Chypriotes grecs, de même que ceux de six étudiants démocrates 
de gauche en Turquie dans les années 1970. L'assassinat de Kutlu Adalı le 6 juillet 1996 est aussi remémoré comme le symbole de la lutte de ce journaliste pour la réunification et l'entente avec les Chypriotes grecs. La gauche conteste la vision nationaliste de «Turcs de Chypre » (Kıbrıs Türkü) dont l'horizon politique serait inévitablement celui $\mathrm{du}$ «turquisme» (Türklük), pour parler au nom des "Chypriotes turcs» (Kıbrısl Türkler). Avec leurs homologues chypriotes grecs, les partis de gauche organisent les événements bicommunautaires qui favorisent le rapprochement, contestant le statu quo et la vision d'une coopération impossible prônée par les nationalistes.

\section{Conclusion : le nationalisme turc face à la concurrence mémorielle}

Les célébrations patriotiques à Chypre du Nord ont donc permis de maintenir une prédominance du nationalisme turc par plusieurs moyens. D'abord, en contribuant à redéfinir les grands événements de l'histoire des Chypriotes turcs suivant une vision nationaliste, le calendrier mémoriel « officiel » défini après 1974 a permis d'entretenir la conflictualité en donnant une image barbare et violente des Chypriotes grecs. De plus, en cherchant à mobiliser activement les citoyens et les écoliers autour de ces célébrations et jours fériés, en évoquant le souvenir des martyrs dans l'espace public et dans les écoles, les nationalistes, en s'appuyant sur un pouvoir autoritaire acquis à leur cause, ont gagné la lutte pour la définition de la réalité sociale durant les années 1980-1990, dans une société isolée et sous embargo économique où la mémoire du conflit demeurait vivace. En tant qu'expressions rituelles de l'historicité d'une nation, les célébrations importées de Turquie ont permis de renforcer le sentiment d'appartenance des Chypriotes turcs à la nation turque et leur identification à un autre État, en stigmatisant l'identité chypriote comme celle des Chypriotes grecs. Enfin, en tant que rituels d'intégration, les célébrations patriotiques ont permis de renforcer la cohésion du groupe nationaliste, son monopole sur l'interprétation de l'histoire et sa domination de l'espace public. Au sud, une vision nationaliste de l'histoire axée sur l'hellénisme, opposée à celle du nationalisme turc, mais dotée d'une structure analogue, a été proposée par le DISY.

Mais nous avons aussi montré l'existence de «contre-mémoires » internes à chacune des communautés, qui contestent les célébrations et les narrations historiques proposées par les nationalistes. Les nationalistes de part et d'autre proposent des récits nationaux fondés sur l'appartenance de Chypre à l'histoire de la Grèce et de la Turquie, en cultivant une vision éminemment conflictuelle du rapport à l'autre. Pour les nationalistes chypriotes grecs, 1974 n'est pas simplement une tragédie parce qu'elle implique la division de l'île, mais symbolise aussi l'échec regretté du projet d'union avec la Grèce. Au contraire, l'intervention militaire turque est présentée par les nationalistes turcs comme le symbole de la fin heureuse des souffrances des années 1960 et comme une promesse de liberté et de construction nationale. Là où les nationalistes célèbrent la mémoire des «Grecs » ou des «Turcs » opprimés par le camp adverse, les démocrates de gauche se remémorent les "Chypriotes " grecs et turcs réprimés par les extrémistes des deux communautés. On voit donc bien comment la mémoire du conflit peut être utilisée politiquement afin d'entretenir la division, mais peut aussi être mobilisée afin de susciter des interprétations plus nuancées de l'histoire favorisant le rapprochement. 
Là où les nationalistes célèbrent la mémoire des « Grecs » ou des « Turcs » opprimés par le camp adverse, les démocrates de gauche se remémorent toutefois les «Chypriotes » grecs et turcs réprimés par les extrémistes des deux communautés. Ces forces politiques et sociales, historiquement minoritaires tant au nord qu'au sud, doivent cependant faire face à une intense propagande d'État et à la diffusion d'une mémoire nationaliste par le biais de l'école et des défilés patriotiques. La mémoire nationaliste est d'autant plus prédominante en RTCN qu'elle se construit sur une intense propagande d'État dans un contexte de démocratisation imparfaite sous la surveillance de l'armée turque, qui joue non seulement un rôle militaire, mais aussi politique, en conditionnant indirectement ce qu'il est possible de dire dans l'espace public sur la mémoire et sur la Turquie. Les célébrations rituelles visent à diffuser une certaine idée de l'histoire des "Turcs de Chypre », de leurs relations idéalisées avec les Turcs et de leurs rapports présentés comme historiquement conflictuels avec les Grecs. Mais la vision du passé de la propagande d'État connaît une crise relative de légitimité depuis une dizaine d'années, tant l'expérience concrète des Chypriotes turcs est éloignée de l'imaginaire politique des nationalistes louant la partition. L'ouverture de nouveaux points de passage le long de la ligne verte, l'insertion de l'île dans les flux migratoires européens et la mondialisation, l'augmentation du niveau de vie des Chypriotes turcs, sont autant de facteurs qui peuvent favoriser une plus grande ouverture et une meilleure connaissance de l'autre. Enfin, la normalisation des alternances politiques depuis 2003 a de même favorisé l'essor d'un nouveau discours d'opposition, et d'une plus grande distance critique des citoyens à l'égard des partis et des discours officiels. C'est pourquoi, malgré la prédominance de la vision mémorielle du nationalisme turc, il est possible de parler de l'essor d'une « concurrence mémorielle », entendue comme le développement de visions distinctes de la mémoire et de l'identité collective des Chypriotes turcs.

\section{BIBLIOGRAPHIE}

ANDERSON Benedict, 2006 [1983], l'Imaginaire national : réflexions sur l'origine et l'essor du nationalisme, Paris : La Découverte.

ATTALIDES Michael, 1979, Cyprus: Nationalism and International Politics, Edinburgh: Q Press.

BACHRACH Peter, BARATZ Morton, 1962, "Two faces of Power", The American Political Science Review, vol. 56, no 4, pp. 947-952.

BAYART Jean-François, 2006 [1989], l'État en Afrique : la politique du ventre, Paris : Fayard.

BLONDY Alain, 1998, Chypre, Paris : Presses universitaires de France.

BOURDIEU Pierre, 1982, Ce que parler veut dire : l'économie des échanges linguistiques, Paris : Fayard.

CANEFE Nergis, 2002, "Citizenship, history and memory in Turkish Cypriot society: Is there room for Cypriotness?", in Christina KoUlouRI (ed.), Clio in the Balkans: The politics of history education, Thessaloniki: Center for Democracy and Reconciliation in Southeast Europe, pp. 383-396. 
CASQUETE Jesus, 2009, En el nombre de Euskal Herria: la religión política del nationalismo vasco radical, Madrid: Tecnos.

COAKLEY John, 2004, "Mobilizing the past: Nationalist images of history", Nationalism and Ethnic Politics, vol. 10, no 4, pp. 531-560.

COPEAUX Étienne, 2002, “Otherness in the Turkish historical discourse: general considerations”, in Christina KOULOURI (ed.), Clio in the Balkans: The politics of history education, Thessaloniki: Center for Democracy and Reconciliation in Southeast Europe, pp. 397-405.

COPEAUX Étienne, MAUSS-COPEAUX Claire, 2005, Taksim! Chypre divisée (1964-2005), Lyon : éditions Aedelsa.

COUfOUdAKIS Van, 1983, “Cyprus”, in MCHALE Vincent (ed.), Political Parties of Europe, Westport: Greenwood Press, pp. 110-125.

DODD Clement, 1993, The Political, Social and Economic Development of Northern Cyprus, Eothen: Hemingford Grey.

GRANDJEAN Geoffrey, JAMIN Jérôme (dir.), 2011, la Concurrence mémorielle, Paris : Armand Colin. HALBWACHS Maurice, 1997 [1925], la Mémoire collective, Paris : Albin Michel.

HOBSBAWM Eric John, 1992, Nations et nationalisme depuis 1780 : programme, mythe, réalité, Paris : Gallimard.

KOLSTO Pal, BLAKKISRUd Helge (eds.), 2004, Nation-Building and Common Values in Russia, Lanham: Rowman and Littlefield.

KOULLAPIS Loris, 2002a, "The presentation of the period 1071-1923 in Greek and Turkish textbooks between 1950-2000", International Textbook Research, vol. 24, no 3, pp. 290-305.

KOULLAPIS Loris, 2002b, “The subject of history in the Greek-Cypriot educational system: A subset of the Greek nation", in Christina KoulouRI (ed.), Clio in the Balkans: The politics of history education, Thessaloniki: Center for Democracy and Reconciliation in Southeast Europe, pp. 407-421.

KIZILYÜREK Niyazi, 2002, "National memory and Turkish-Cypriot textbooks", in Christina KOULOURI (ed.), Clio in the Balkans: The politics of history education, Thessaloniki: Center for Democracy and Reconciliation in Southeast Europe, pp. 432-435.

LAVABRE Marie-Claire, 2000, «Usages et mésusages de la notion de mémoire », Critique Internationale, $\mathrm{n}^{\circ} 7$, p. 48-57.

MAVRATSAS Caesar, 1997, “The ideological contest between Greek-Cypriot nationalism and Cypriotism 1974-1995: Politics, social memory and identity", Ethnic and Racial Studies, vol. 20, no 4, pp. 717-737.

MILLAS Hercules, 1991, "History textbooks in Greece and Turkey”, History Workshop Journal, vol. 31, no 1, pp. 21-33.

MORAG Naday, 2004, "Cyprus and the clash of Greek and Turkish nationalisms", Nationalism and Ethnic Politics, vol. 10, no 4, pp. 595-624.

NAVARO-YASHIN Yael, 2012, The make-believe space: Affective geography in a postwar polity, Durham and London : Duke University Press.

NORA Pierre, 1984, les Lieux de mémoire, t. I, la République, Paris : Gallimard. 
NORA Pierre, 1978, «La mémoire collective », in Jacques LE GOFF (dir.), la Nouvelle Histoire, Paris : Retz-CEPL, p. 398-401.

OLSON Mancur, 2011 [1971], Logique de l'action collective, Bruxelles : Presses de l'université de Bruxelles.

PAPADAKIS Yannis, 2008, History Education in Divided Cyprus: A comparison of Greek Cypriot and Turkish Cypriot schoolbooks on the "History of Cyprus", PRIO Report, no 2, pp. 10.

PAPADAKIS Yannis, 2003, "Nation, narrative and commemoration: Political ritual in divided Cyprus", History and Anthropology, vol. 14, n 3, p. 259-275.

PAPADAKIS Yannis, 1998, “Greek Cypriot narratives of history and collective identity: Nationalism as a contested process", American Ethnologist, vol. 25, no 2, pp. 145-161.

PAPADAKIS Yannis, 1993, "The politics of memory and forgetting in Cyprus", Journal of Mediterranean Studies, vol. 3, no 1, pp. 135-153.

PETITHOMme Mathieu, 2015, «Commémorer les gudaris d'hier pour légitimer la violence d'aujourd'hui : une étude iconographique du détournement du Bizkargi et de l'Albertia Eguna au Pays basque ", Pôle Sud : revue de science politique de l'Europe méridionale, n 42/1, p. 105-135.

POST, 2007, “Textual and visual analyses of lower secondary school history textbooks:

Comparative analysis of the old and the new textbooks", Lefkoşa.

THEOPHYLACTOU Demetrios, 1995, Security, identity and nation-building: Cyprus and the European Union, Avery: Aldershot.

UĞURAL Çetin, SOYER Derdi S., 1998, şükran Ekonomisi: Üzerimizdeki Ipotek (Kuzey Kıbrıs Ekonomisi Bütçe Analizleri), Nicosia: Naci Talat Vakfi Yayınları.

VURAL Yucel, ÖZUYANIK Evrim, 2008, "Redefining identity in the Turkish-Cypriot school History textbooks: A step towards a united Cyprus", South European Society and Politics, vol. 13, no 2, pp. 133-154.

WHITE Hayden, 1978, Tropics of Discourse: Essays in Cultural Criticism, Baltimore/London: John Hopkins University Press.

ZEKI Serter Vehbi, 2002, Kıbrıs Tarihi: orta I, II, III: Sınıflar için [Histoire de Chypre: niveaux I-II-III du collège], Lefkoşa : publication du ministère de l'Éducation nationale.

ZEKI Serter Vehbi, FIKRETOĞLU Ozkan, 2002, Kıbrıs Türk Mücadele Tarihi : Lise I, II, III [Histoire de la lutte des Turcs de Chypre], Lefkoşa : publication du ministère de l'Éducation nationale.

\section{NOTES}

1. Entretien avec Omer Adal (DP), 49 ans, rue Server Somuncuoğlü, 6 février 2012. L'ensemble des entretiens cités ci-dessous a été réalisé à Lefkoşa, Nicosie-nord.

2. Entretien avec Cüneyt Küçük (UBP), 33 ans, rue Sarayönü, 6 février 2012.

3. Déclaration de Rauf Denktaş, BRT 1, 30 juin 2002, cité par Navaro-Yashin, 2012, p. 52.

4. Entretien avec Kemal, 31 ans, militaire turc, place de Kyrenia, 10 février 2012.

5. Expression reprise d'un entretien avec Alper Hasta, 39 ans, secrétaire général des Loups Gris (Ülkü Ocaklari), avenue Mehmet Akif, 15 février 2012. 
6. Entretien avec Mehmet Enginar, 28 ans, avenue Mehmet Akif, 15 février 2012.

7. Entretien avec Huseyin, 25 ans, militaire, porte de Kyrenia, 5 février 2012.

8. Entretien avec Alpay Durduran, 70 ans, (YKP), rue Tanzimat, 14 février 2012.

9. Entretien avec Izzet Izcandir, secrétaire général du parti de Chypre unifié (Birleșik Kıbrıs Partisi, BKP), 20/2/2012.

10. Entretien avec Saffet Nadiri, membre du bureau du parti de l'unité nationale (Ulusal Birlik Partisi, UBP), rue Sarayönü, 6/2/2012.

11. Cyprus Daily News, $3 / 5 / 2014$.

12. Entretien avec Murat Kanatli, rue Tanzimat, 14/2/2012.

13. "Atun: EOKA Idealleri Karşisında Bugün de Siperlerdedir», Kıbrıs Gazetesi, 2 avril 1997.

14. "Demonstrations to remember December 1963 events", Cyprus Mail, 22 décembre 2013.

\section{RÉSUMÉS}

Cet article étudie l'utilisation politique des célébrations patriotiques et des manuels scolaires par le nationalisme turc à Chypre du Nord. Il montre que depuis la partition de 1974, un calendrier mémoriel a été utilisé pour diffuser un nationalisme d'État proposant une narration simplifiée de l'histoire du conflit, glorifiant le rôle de la Turquie et stéréotypant la violence des Chypriotes grecs, avec lesquels la coexistence pacifique serait impossible. En entretenant la mémoire des martyrs, les nationalistes turcs ont imposé un discours politique idéologisant et conflictuel fondé sur la peur et la menace pour justifier leur lutte de libération nationale et l'indépendance de la RTCN. Un discours analogue, fondé sur l'appartenance de Chypre à l'hellénisme et la résistance au colonisateur britannique et à la Turquie, domine l'espace politique chypriote grec. Mais des contre-mémoires internes existent aussi au sein de chaque communauté, et proposent des interprétations moins essentialistes et plus nuancées de l'histoire du conflit.

This article studies the political usages of patriotic celebrations and school textbooks by Turkish nationalism in Northern Cyprus. It shows that since the division in 1974, a memorial calendar has been used to diffuse a state nationalism that proposes a simplified narrative of the history of the conflict. Such narrative glorifies the role of Turkey and provides stereotypes on the violence of Turkish Cypriots, inducing that peaceful coexistence would be impossible. By celebrating the memory of the martyrs, Turkish nationalists have imposed an ideological and conflicting political discourse based on the feelings of fear and threat to justify their national liberation struggle and TRNC's independence. An analogous discourse based on the belonging of Cyprus to Hellenism and the resistance against British colonial rule and Turkey dominates the Greek Cypriot political space. But alternative internal memories also exist within each community, and propose less essentialist and more nuanced interpretations of the history of the conflict.

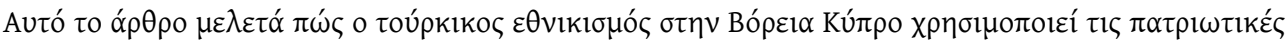

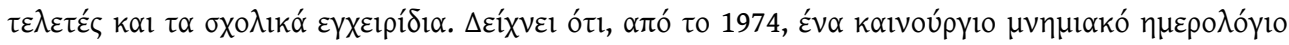

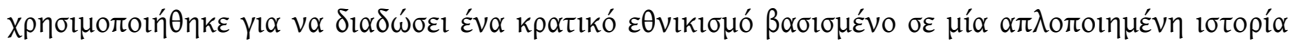




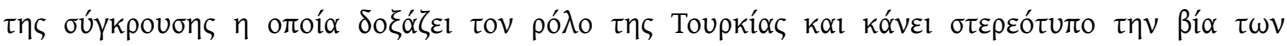

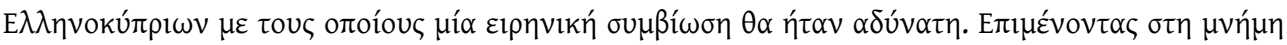

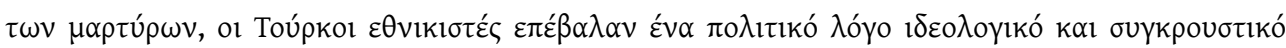

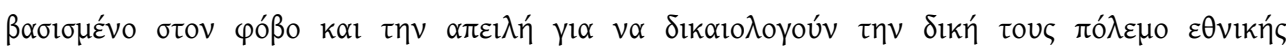

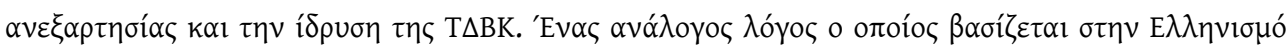

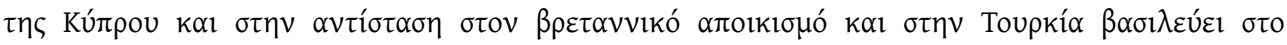

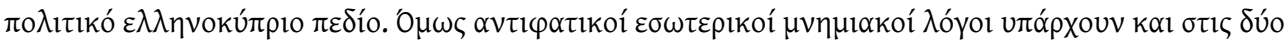

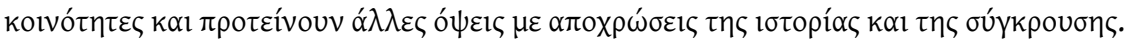

\section{INDEX}

Mots-clés : nationalisme, nationalisme, mémoire, mémoire, politique, politique motsclesmk НАЦИОНАЛИЗМОТ, МЕМОРИЈА, ПОЛИТИКА, КИПАР, СЕВЕРНИОТ ДЕЛ НА КИПАР, 1974-2014, ДВАЕСЕТ И ПРВИОТ ВЕК, СОЦИЈАЛНА И ПОЛИТИЧКА ИСТОРИЈА

Keywords : Nationalism, Memory, Politics, Northern Cyprus, 1974-2014, Twenty first century, Social and political History

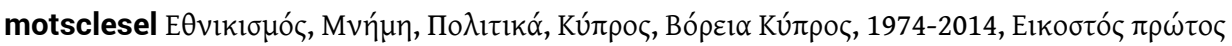

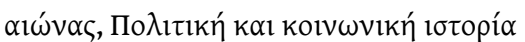

Thèmes : Histoire sociale et politique, Sociologie politique

motsclestr Milliyetçilik, Bellek, Politika, Kıbrıs, Kuzey Kıbrıs, 1974-2014, Yirmi birinci yüzyılın, sosyal ve siyasi tarihi

Index géographique : Chypre, Chypre du Nord

Index chronologique : Chypre du Nord -- 1974-2014

\section{AUTEUR}

\section{MATHIEU PETITHOMME}

Maître de conférences en science politique IUT de Besançon/CRJFC 